\title{
ShiverPaD: A Glass Haptic Surface That Produces Shear Force on a Bare Finger
}

\author{
Erik C. Chubb, Student Member, IEEE, J. Edward Colgate, Member, IEEE, and \\ Michael A. Peshkin, Member, IEEE
}

\begin{abstract}
We discuss the design and performance of a new haptic surface capable of controlling shear force on a bare finger. At the heart of the ShiverPaD is the TPaD variable friction device. It modulates the friction of a glass surface by using $39 \mathrm{kHz}$ out-of-plane vibrations to reduce friction. To generate shear forces, the TPaD is oscillated in-plane (i.e., "shivered") while alternating between low and high friction within each cycle. In previous research, the ShiverPaD produced shear forces using in-plane vibrations below $100 \mathrm{~Hz}$. In this research, we develop a new ShiverPaD that produces force using $854 \mathrm{~Hz}$ vibrations, where human sensitivity to vibration is diminished. The new device is used to display a virtual toggle switch and a variety of virtual edges. A human subject study is conducted to demonstrate that users can easily trace virtual edges displayed on the surface of the ShiverPaD.
\end{abstract}

Index Terms-Haptics, tactile interface, variable friction.

\section{INTRODUCTION}

$\mathrm{T}$ $\mathrm{HE}$ increasing prevalence of touch pad and touch screen interfaces in cars, kiosks, personal computers, and mobile devices has led to growing interest in haptic interaction associated with touch-input devices. The human fingertip is well suited to touching, manipulating, sensing, and perceiving on surfaces. The challenge has been to create interface modalities that exploit natural human fingertip capabilities, but are also flexible and programmable. Here, we use the term "haptic surface" to describe any device that can display virtual effects on a physical surface. We review some of the previous work in "surface haptics" and then present our contribution, the ShiverPaD.

\subsection{Vibrotactile Displays}

Perhaps the most basic and ubiquitous haptic surface is a cell phone vibrated by an eccentric-mass motor. The vibrating surface can communicate a few bits of information about incoming calls and button press confirmation. Kim et al. broadened this concept by using piezoelectric actuation to present nonsinusoidal profiles [1]. Others have developed similar haptic interfaces but with different actuation techniques [2], [3].

Poupyrev and Maruyama [4] introduced a handheld device with piezoceramic actuators placed at the corners of the touch screen. They indicate that a single-cycle sinusoid can create the haptic illusion of the "click" of a button. Artificial Muscle Inc. has demonstrated a similar device, but using dielectric elastomer actuation [5].

- E.C. Chubb is with the Northwestern University, 5925 Seville Circle, Orchard Lk, MI 48324. E-mail: erikchubb@gmail.com.

- J.E. Colgate and M.A. Peshkin are with the Department of Mechanical Engineering, Northwestern University, 2145 Sheridan Rd, Evanston, IL 60208. E-mail: \{colgate, peshkin\}@northwestern.edu.

Manuscript received 28 Aug. 2009; revised 13 Dec. 2009; accepted 20 Jan. 2010; published online 18 Mar. 2010

Recommended for acceptance by L. Jones, M. Harders, and Y. Yokokohji.

For information on obtaining reprints of this article, please send e-mail to: toh@computer.org, and reference IEEECS Log Number THSI-2009-08-0063. Digital Object Identifier no. 10.1109/ToH.2010.7.

$1939-1412 / 10 / \$ 26.00$ (C) 2010 IEEE

\subsection{Shape Changing Surfaces}

Another category of haptic surfaces can alter their shape. A dynamic braille display is a classic example. Yang et al. created a $6 \times 5$ pin array actuated by piezoelectric bimorphs, capable of $700 \mu \mathrm{m}$ displacement [6]. Their haptic experiments proved that edge shape could easily be communicated through the array. Kato et al. reduced the space needed for such a device by creating a $1 \mathrm{~mm}$ thick film capable of displaying braille [7].

The STReSS tactile display developed by Pasquero and Hayward uses a $10 \times 10$ array of piezoelectric actuated contactors that move laterally to create compressive and tensile strains in the skin of the fingertip [8]. This device can apply tensile and compressive stresses to different areas of the fingertip simultaneously. Levesque and Hayward's research indicates that the fingertip experiences similar stress distribution when it encounters edges or small bumps [9].

On a larger scale, Harrison and Hudson [10] created a device that can "pop out" a preset array of buttons for commonly used features (such as an alphanumeric keyboard) and then merge them back into the plane when the display is being used as a continuous, flat touch screen.

\subsection{Variable Friction Devices}

A third category of haptic surfaces relies on the principle that lateral forces can be used to create the illusion of texture and surface features. This idea was originated by Minsky [11] and furthered by Robles-De-La-Torres [12] and Hayward, both of whom worked with kinesthetic displays. It has served as inspiration, however, for a number of variable friction devices.

Yamamoto et al. [13] created a display that uses electrostatics to control frictional forces on the fingertip. The user rests his finger on a thin-film slider (or thimble) and the frictional forces between the slider and the substrate are controlled. Takasaki et al. [14] created a transparent device with a similar slider-based interface, except that friction modulation was achieved through $15 \mathrm{MHz}$ surface acoustic 
waves. The vibration created a vertical motion on the order of $10 \mathrm{~nm}$ that resulted in periodic contact with the hard spheres on the underside of the slider. The periodic contact reduced the perceived friction. A disadvantage to these devices is that they do not operate on a bare finger.

\subsubsection{The TPaD Variable Friction Device}

Watanabe and Fukui [15] developed the first ultrasonic vibrating plate capable of controlling the surface roughness displayed to a bare finger using the squeeze film effect. A squeeze film is high pressure air that forms between two relatively flat surfaces when one is vibrated at high frequency. If the high pressure overcomes the normal force, then the objects will be separated by a "cushion" of air. The $76 \mathrm{kHz}$ vertical motion in Watanabe's device created a squeeze film of air that was shown to mask the roughness of fine-grit sand paper. Biet et al. [16] used an array of piezoelectric actuators glued to the underside of a metallic sheet. They measured the amplitude of the out-of-plane vibrations to be about $1 \mu \mathrm{m}$. Frictional forces can be modulated continuously by adjusting the amplitude of the vibration.

In a similar approach, the Tactile Pattern Display (TPaD) variable friction device, is composed of a piezoelectric actuator glued to the underside of a glass plate [17]. There is convincing preliminary evidence that a user actively exploring the surface of a TPaD can experience the haptic illusion of textures and surface features, such as smooth bumps.

One disadvantage of all variable friction devices is that they can only produce force antiparallel to the direction of motion.

\subsection{Tractive Devices}

Again leveraging the idea that lateral forces can be used to create the illusion of surface features, Wang et al. introduced a Haptic Overlay Device (HOD) for touch screens [18], [19]. The user operates the HOD by bringing a finger into contact with the overlay material. The overlay is connected to drive rollers which can translate the overlay and create surface haptic effects. One difficulty with this device is that the overlay must be made significantly larger than the touch surface to prevent it from running out of travel. For the same reason, the display must be quickly returned to the home position after the finger is lifted. With the ShiverPaD, we hope to display haptic effects similar to the HOD, but without the issues associated with limited travel.

\subsection{The ShiverPaD: A New Haptic Surface}

The ShiverPaD is a device comprised of a TPaD (discussed in Sections 1.3.1 and 2) and the mechanisms required to "shiver" it in-plane. It creates a net force on a finger by alternating between low and high friction at the same frequency that the TPaD is oscillated in-plane. During each low-friction phase, the TPaD slips beneath the finger without applying significant force. During each high-friction phase, an impulse is provided to the finger. The time average of these impulses creates a nonzero net force. In Fig. 1, the process for creating a rightward net force is illustrated.

The original ShiverPaD (introduced in [20]), used vibrations below $100 \mathrm{~Hz}$, which created a strong "vibration artifact" in addition to the net force. In an effort to reduce

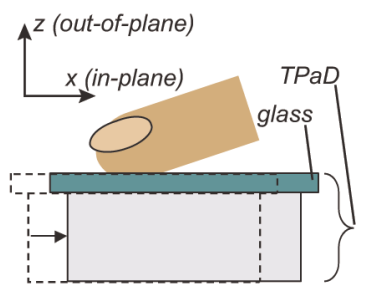

(a)

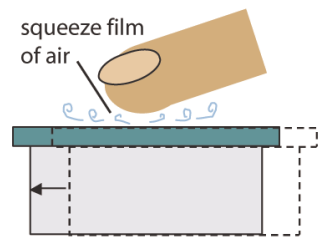

(b)
Fig. 1. The method for generating a rightward net force. (a) Rightward movement with high friction. (b) Leftward movement with low friction.

this artifact, we have increased the frequency. As predicted by Bolanowski et al. [21], we experienced peak sensitivity around $300 \mathrm{~Hz}$, and consequently moved to higher, less perceptible, frequencies. The ShiverPaD, described in this paper, runs at $854 \mathrm{~Hz}$. The perception of vibrations has not completely disappeared, but is significantly reduced compared to the previous device.

Like the Haptic Overlay Device discussed above, the ShiverPaD can actively apply force to a finger regardless of the direction of finger motion. This is in contrast to variable friction devices that can only produce forces antiparallel to the direction of finger motion. Unlike the HOD, the ShiverPaD can do this without the issue of running out of travel. Like pin-array-type shape changing surfaces, the ShiverPaD is capable of creating the haptic illusion of edges and surface features, but can be made transparent and does not require the mechanical complexity.

In this paper, we discuss the design and performance of a 1DOF ShiverPaD (Sections 2 and 3), demonstrate some virtual environments (Sections 4 and 5), and report on a short human subject study (Section 6) to show that users can easily find and trace virtual edges displayed on the ShiverPaD.

\section{The ShiverPaD Device}

The four components of our ShiverPaD prototype are labeled in Fig. 2, and defined here,

1. The voice coil provides the actuation for the inplane, or "shiver," motion.

2. The linear slide ensures that the motion is constrained to the $x$-direction.

3. The "shiver spring" together with the mass of the $\mathrm{TPaD}$ creates a simple harmonic oscillator. The amplification at resonance is leveraged to achieve greater lateral oscillation amplitudes.

4. The TPaD is a variable friction display capable of altering friction on the millisecond time scale or better.

The final component of the ShiverPaD (not shown in Fig. 2) is the finger position sensor. This laser-based system is capable of reporting both $x$ and $y$ finger positions. The construction and capability of the finger positioning sensor are discussed further in Section 2.1.1.

The voice coil provides sinusoidal forcing at the resonance of the system causing the $\mathrm{TPaD}$ to oscillate in the $x$-direction at $854 \mathrm{~Hz}$ with an amplitude of approximately $\pm 20 \mu \mathrm{m}$. The voice coil is an Equipment Solutions VCS10H, capable of 6.7 N max continuous force and $25 \mathrm{~W}$ max continuous power, 


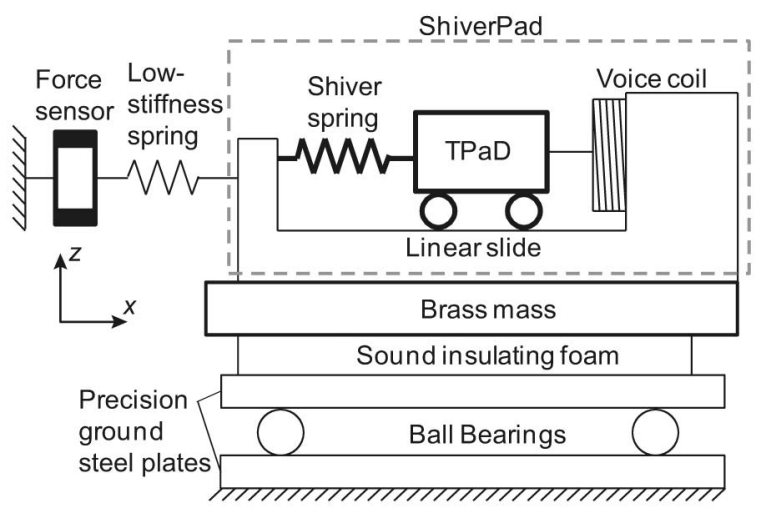

Fig. 2. Conceptual schematic of the ShiverPaD. The purpose of the components outside of the dashed gray rectangle is to measure the forces applied to the finger.

but most experiments were run at approximately 70 percent of maximum power. The position of the TPaD is measured by a submicron resolution linear displacement sensor integral to the voice coil. This displacement sensor was used only for postprocess analysis, and is not necessary for the real-time control of the ShiverPaD.

The value of $854 \mathrm{~Hz}$ was chosen because the combination of moving mass and structural compliance did not permit a significantly higher resonant frequency, regardless of the choice of shiver spring. Using the mass and natural frequency, it can be calculated that the combined stiffness of the shiver spring in series with the spring attachment structure is $1.8 \mathrm{kN} / \mathrm{mm}$.

Friction is modulated on the glass surface of the TPaD by applying a $39 \mathrm{kHz}$ sinusoidal voltage to the piezoelectric element mounted on the underside of the glass. The $39 \mathrm{kHz}$ signal is generated by a AD9833 waveform generator chip and amplified to $\pm 40 \mathrm{~V}$ using an audio amplifier. When applied to the piezoelectric, it causes resonant vibrations of the glass plate. As discussed earlier, these vibrations produce a squeeze film of air underneath the fingertip, leading to a reduction of friction. Winfield et al. [17] found that at high excitation voltages, the friction between the glass and a finger is approximately $\mu=0.15$, while at zero voltage, the surface has the friction of normal glass $(\mu \approx 0.95)$.

The purpose of the remaining components illustrated in Fig. 2 is to measure the force applied to the finger. The entire ShiverPaD assembly is placed on an ultralow friction sled. The sled consists of three steel balls sandwiched between two precision-ground steel plates. When the ShiverPaD applies force to the finger, the reaction force that keeps the sled from moving is supplied through the force sensor. Therefore, the DC component of the force sensor reading is equivalent to the DC force on the fingertip. The force sensor is a $1 \mathrm{DOF}$ tension/compression load cell with $\pm 250 \mathrm{~g}$ range. The brass mass and the low stiffness spring shown in Fig. 2 act as a mechanical low-pass filter to the force signal. Since their resonant frequency is approximately $13 \mathrm{~Hz}$, force readings with frequency content well below $13 \mathrm{~Hz}$ are reliable.

The brass mass serves a second function. Since the lower half of the ShiverPaD is not grounded, it oscillates in reaction to the upper half oscillating. The brass mass ensures a high ratio between the lower mass $(2.4 \mathrm{~kg})$ and the upper mass

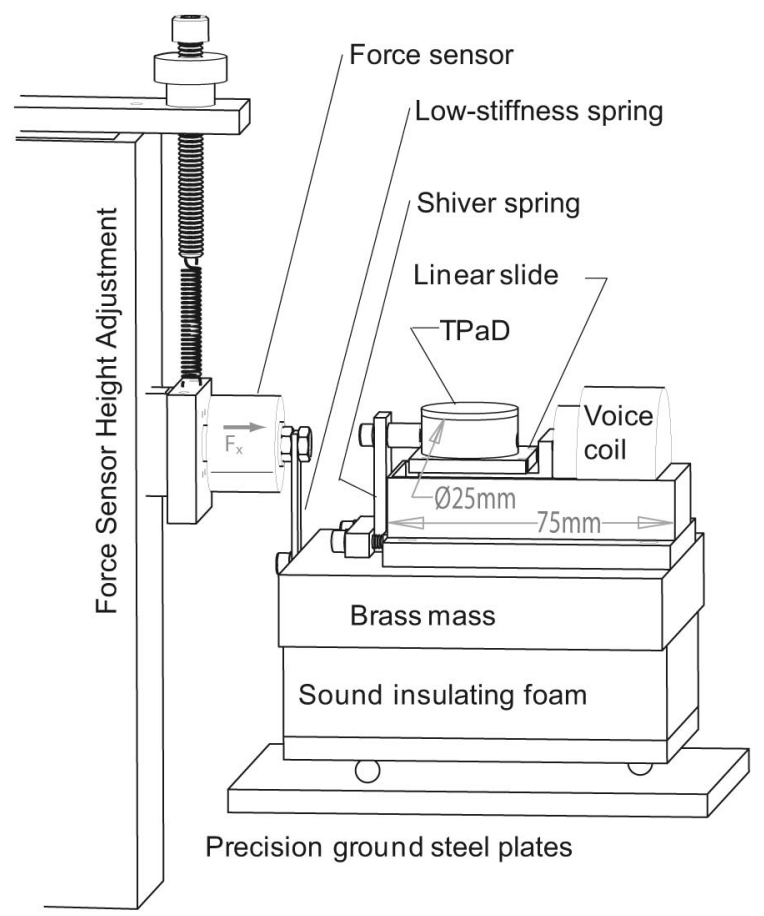

Fig. 3. The ShiverPaD device and the force sensing system.

$(0.063 \mathrm{~kg})$. Therefore, the amplitude of vibration of the upper half is 38 times greater than the lower half, making the lower half's vibrations negligible.

Fig. 3 is a line drawing of the actual device. Here, it can be seen that the conceptual springs shown in Fig. 2 are actually leaf springs. The natural frequencies of the system can be adjusted by changing the thickness of the leaf spring. Also note that the force sensor height is adjustable and compliant in order to reduce off-axis loads on the force sensor.

Since the device vibrates at $854 \mathrm{~Hz}$, it emits audible sound at the same frequency. The foam sandwiched between the mass and the sled reduces the transmission of vibration to the table and the surroundings.

The ShiverPaD in Fig. 3 has significant surface area transverse to the axis of shiver motion. Like a speaker, the transverse area is effective at radiating sound. Ear protection is not needed, but lengthy exposure is tiresome. During the human subject trails (Section 6), the subjects were offered the use of ear protection. All subjects declined.

We predict that the sound volume could be significantly reduced by minimizing the transverse area of the vibrating parts. Since the parts set into shiver motion could theoretically be reduced to a sheet of glass, a piezoelectric, and a miniature actuation device, it is conceivable that the transverse area could be reduced by an order of magnitude or more.

\subsection{Electronics and Control}

A PIC chip used for controlling the ShiverPaD generates the $854 \mathrm{~Hz}$ sinusoidal signal for the voice coil, and issues the command to the waveform generator to start/stop the $39 \mathrm{kHz}$ signal. Since it provides both functions, it can dictate the phase relationship between the TPaD's friction 


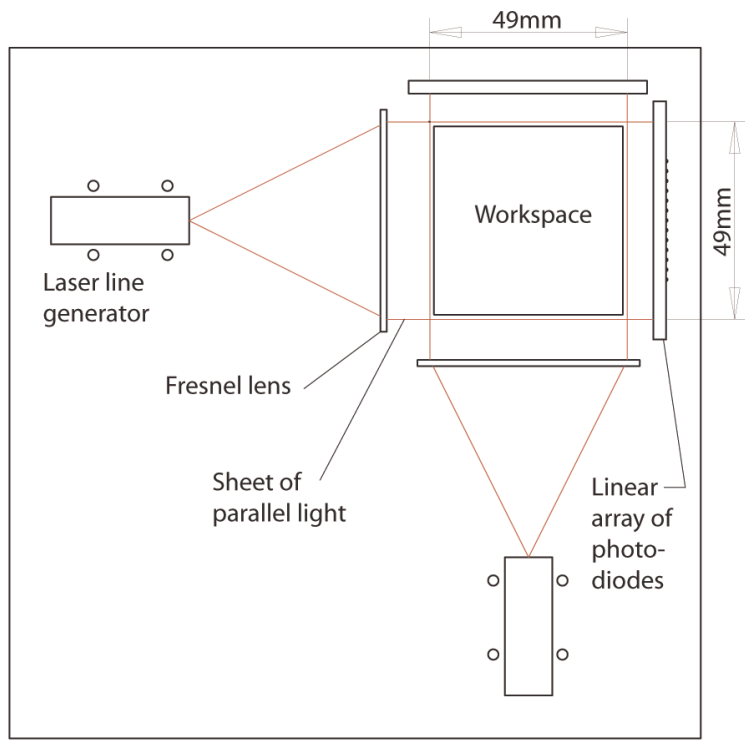

Fig. 4. The laser-based Finger Positioning System.

level and the lateral motion. The PIC's loop-time is not constant, and depends on the length of the code branches executed, but is typically less than $60 \mu \mathrm{s}$.

The output of a laser-based finger positioning system (Section 2.1.1) is passed to the control algorithm in the PIC chip. The force applied to the finger can be altered in real time based on the finger position.

\subsubsection{The Finger Positioning System}

At any instant in time, the entire ShiverPaD surface is forcing in one direction and magnitude. In other words, it displays a spatially constant force field. To create the illusion of a spatially varying force fields, finger position is used to vary force as a function of location.

The finger positioning system for the ShiverPaD must have high spatial resolution to ensure that virtual environments can be consistently rendered. Commercially available IR devices had resolutions too low to be useful, therefore, we developed the laser-based finger positioning system shown in Fig. 4. It has a workspace of $49 \mathrm{~mm} \times 49 \mathrm{~mm}$, an update rate of $2 \mathrm{~ms}$, and a resolution around $0.1 \mathrm{~mm}$. The workspace of the finger positioning system is placed $2 \mathrm{~mm}$ above and parallel to the ShiverPaD's glass surface.

Each laser line generator creates a planar "fan" of laser light. The cylindrical Fresnel lens redirects the divergent fan of light into a sheet of parallel light which travels just above the haptic surface and strikes a linear photodiode array.

A dedicated PIC chip is used to acquire and analyze the data from the linear photodiode array (TAOS TSL1406R). The light intensity at each pixel along the linear array is transmitted to the dedicated PIC as an analog value. The PIC calculates the centroid of the finger's shadow and outputs an analog voltage proportional to the finger position, which is read by the ShiverPaD's primary control PIC.

\subsubsection{Achieving Fast Transitions between Low and High-Friction Levels}

To create a net force, the transition between friction levels must occur in much less than one half of a shiver oscillation. Therefore, as shiver frequency increases, both

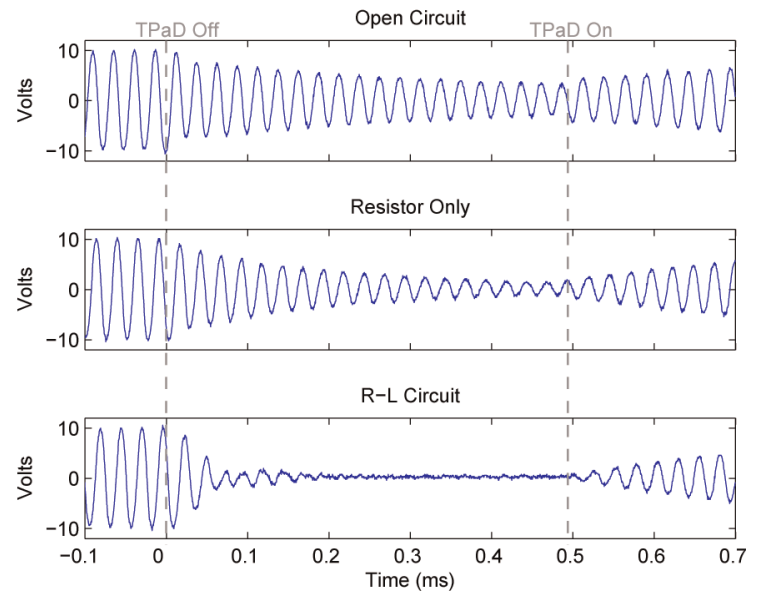

Fig. 5. The effect of the damping circuit on the unforced ultrasonic $\mathrm{TPaD}$ oscillations. The oscillations are damped by the "resistor only" circuit, and even more heavily damped by the R-L circuit.

the low-to-high and high-to-low friction transition speeds must increase similarly. We were able to improve the low-to-high friction transition with the addition of electrical damping. We accomplished this by connecting a tuned inductor-resistor circuit to the piezoelectric actuator [22], [23]. With this circuit, the ring-down time of the TPaD glass was reduced by a factor of 7 .

By intermittently connecting the circuit, we are able to increase the damping during the ring-down period, while leaving it unaffected otherwise. The increase in damping improved the rate of transition from low-to-high friction, without affecting the amplitude or energy consumption during the low-friction phase. The circuit is described, in detail, in [24].

To determine the efficacy of the inductor-resistor network, we ran tests on three different systems.

1. In the Open Circuit (or baseline) condition, the $\mathrm{TPaD}$ glass is allowed to ring down without control.

2. In the Resistor Only condition, an optimally tuned resistor is switched in and out of the circuit.

3. In the R-L Circuit condition, the full R-L circuit is switched in and out of the circuit.

The plots in Fig. 5 demonstrate how the three different damping methods affect the decay of the TPaD oscillations. The amplitude data are the voltage generated by a second, smaller piezoelectric glued to the glass, and used exclusively for sensing. These data comprise a little less than a full shiver cycle ( $854 \mathrm{~Hz}$ vibration in the $x$-direction), but it is possible to see one instance of the TPaD turning on and one instance of it turning off-these time points are indicated.

Adding the inductor-resistor network was found to improve the ShiverPaD's ability to generate net force at the fingertip by 31 percent at $854 \mathrm{~Hz}$ [24]. All of the data presented in this paper use this optimized R-L circuit to improve the peak force capability.

It should be noted that the data in Fig. 5 are taken without a finger in contact with the TPaD. As the normal force between the finger and the TPaD increases, the damping of the ultrasonic vibrations increases. At especially high normal forces, the net effect of the R-L circuit maybe reduced below 31 percent. 


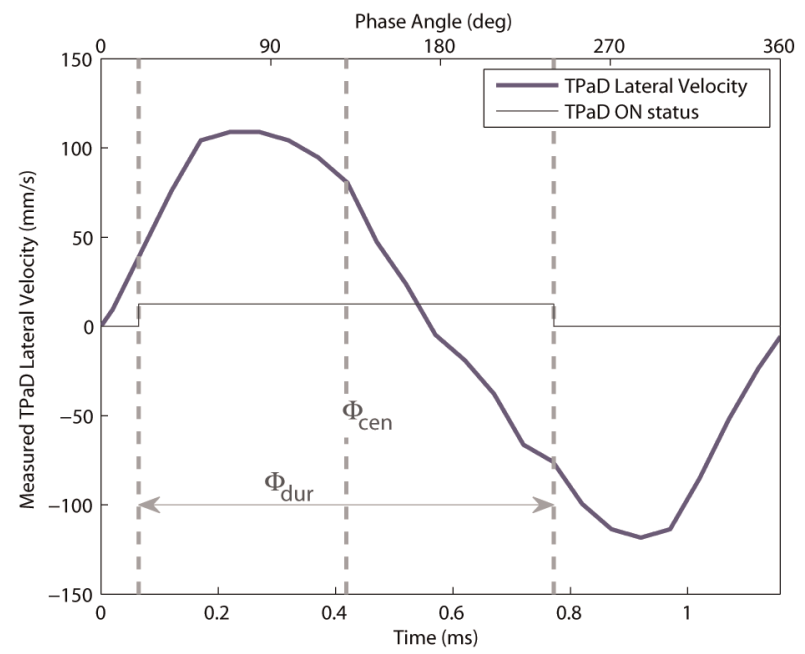

Fig. 6. $\Phi_{d u r}$ is the fraction of the cycle when the TPaD is on. $\Phi_{c e n}$ is the phase angle of lateral velocity half way between the TPaD on and off points. In this example, the TPaD turns on at 20 degrees and remains in the on state for a little over half of the cycle $\left(\Phi_{d u r}=220^{\circ}\right)$ and turns off at 240 degrees. Therefore, $\Phi_{c e n}=130^{\circ}$.

\section{Characterization of Force Generation}

As described in Section 1.5, to produce a net force, the TPaD is set to high friction while its velocity is in one direction and set to low friction when its velocity is in the other direction. Here, we broaden that concept and demonstrate that by changing the duration and phasing of the TPaD on/off signal, we are able to control the direction and magnitude of the net force.

\subsection{Definition of $\Phi_{d u r}$ (Duration) and $\Phi_{c e n}$ (Phasing)}

Changing the fraction of the cycle for which the TPaD is on changes the magnitude of the net force. We define $\Phi_{d u r}$ as the fraction of the full cycle where the TPaD is on (low friction), and measure it in degrees. It is defined graphically in Fig. 6.

Changing the phase delay between the lateral velocity and the $\mathrm{TPaD}$ on/off signal can change both the direction and magnitude of the net force. In this paper, we use it only to change the force direction. It is most convenient to characterize the phase delay by the phase angle of lateral velocity at the center point of the TPaD-on pulse. This point is defined as $\Phi_{c e n}$, and is also defined in Fig. 6.

\subsection{The Effect of Phasing and Duration on Force}

We hypothesize that maximum force will be produced if the squeeze film is present when the lateral velocity of the glass surface is positive and absent when the lateral velocity is negative (or vice versa). If there are no delays in the system, we would expect that $\left(\Phi_{c e n}, \Phi_{d u r}\right)=\left(90^{\circ}, 180^{\circ}\right)$ would produce the greatest rightward force, and $\left(270^{\circ}, 180^{\circ}\right)$ would produce the greatest leftward force.

To determine how duration and phase affect the magnitude of the net force, $256 \times 25$ points were tested in the $\Phi_{c e n}-\Phi_{d u r}$ domain. Fig. 7 summarizes the data. To determine if the $\mathrm{TPaD}$ was in the high or low-friction state, voltage from a dedicated sensing piezoelectric was rectified and then a threshold applied. This is advantageous because the sensing piezoelectric only produces a voltage once the

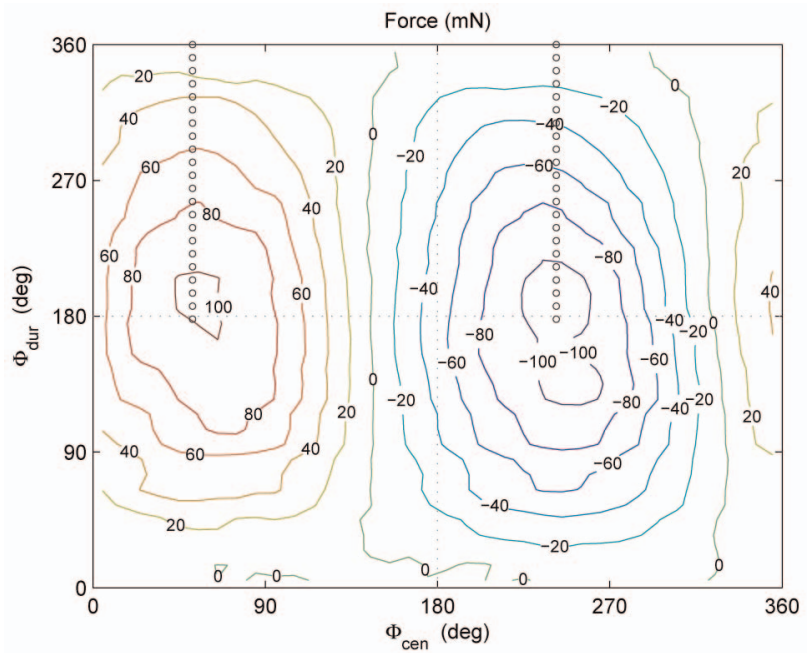

Fig. 7. The contours are the equal force lines measured in $\mathrm{mN}$. Peak force values occur near $\Phi_{d u r}=180^{\circ}$. As $\Phi_{c e n}$ is changed, the net force shifts from rightward to leftward and back again. The thick dotted lines indicates the subset of the $\Phi_{c e n}-\Phi_{d u r}$ domain used in the control strategy.

TPaD is truly oscillating. Therefore, the $\Phi_{c e n}$ and $\Phi_{d u r}$ values reported are free from the influence of both control latencies and $\mathrm{TPaD}$ dynamics.

As expected, when the $\mathrm{TPaD}$ is in its high friction state for the whole cycle $\left(\Phi_{d u r}=0^{\circ}\right)$, zero force is produced because the finger is pushed leftward as much as it is pushed rightward. Similarly, when the $\mathrm{TPaD}$ is in its low-friction state for the whole cycle $\left(\Phi_{d u r}=360^{\circ}\right)$, the squeeze film isolates the finger from the lateral movement of the TPaD for the entire cycle and no appreciable force is applied to the finger. As predicted, the peak force values occur when duration is near half of the full cycle $\left(\Phi_{d u r}=180^{\circ}\right)$.

Moving along the $x$-axis of Fig. 7 , we see that the optimum leftward force is produced at $\Phi_{c e n} \approx 50^{\circ}$, which is 40 degrees less than expected. In fact, the whole surface is shifted 40 degrees in the negative direction. We speculate that the need for this phase advance compared to our expectations is due to the time required for the squeeze film to form or decay.

\subsection{The Control Strategy}

To control the force produced by the ShiverPaD in real time, it is best to create a mapping from the desired force to the values of $\Phi_{c e n}$ and $\Phi_{d u r}$ that produce it. To create maximum magnitude forces, we use the peak points from Fig. 7.

To create zero force, we get to choose among the many possible zero-valued points in the $\Phi_{c e n}-\Phi_{d u r}$ domain. By choosing $\Phi_{d u r}=360^{\circ}$ to create zero force, the squeeze film continually isolates the user from the underlying shiver vibrations making them nearly unnoticeable.

Joining the peak and zero points creates the subset of the $\Phi_{c e n}-\Phi_{d u r}$ domain we use to control the direction and magnitude of force. The subset is denoted by the dotted line in Fig. 7.

To keep the real-time code simple, we use linear interpolation between the peak and zero force values. Fig. $8 \mathrm{a}$ is the mapping used to translate the virtual environment force requests to the low-level ShiverPaD control parameters, $\Phi_{c e n}$ and $\Phi_{d u r}$. Fig. 8b is the actual force produced by this mapping. 


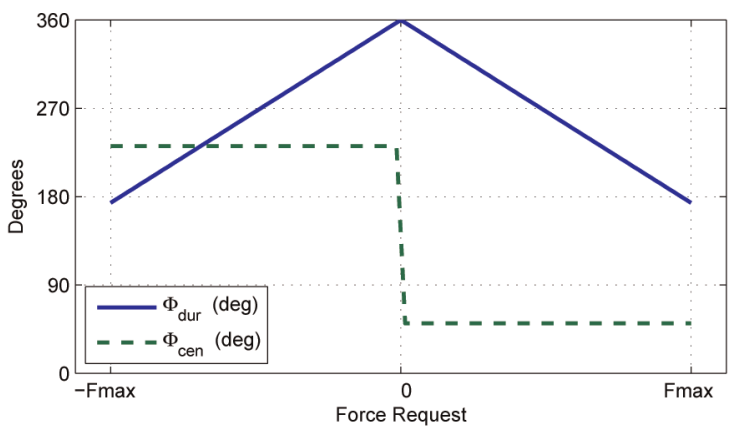

(a)

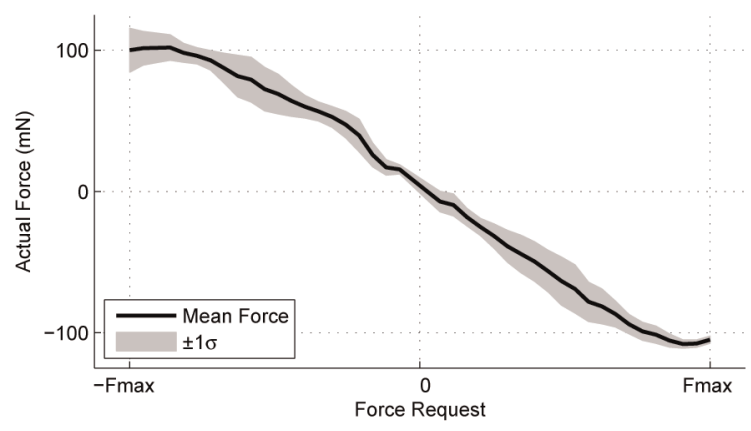

(b)

Fig. 8. The control strategy and the actual force it produces. (a) The mapping used to translate a force request to the low-level ShiverPaD control parameters, $\Phi_{c e n}$ and $\Phi_{d u r}$. When these values of $\Phi_{c e n}$ and $\Phi_{d u r}$ are plotted against each other they form the subset indicated in Fig. 7. (b) The actual forces produced when the mapping in (a) is used. The Mean Force is extracted from the data in Fig. 7 by taking the values along the subset used for control.

In summary, as the finger moves through a virtual environment, $\Phi_{c e n}$ takes on one of two discrete values depending on whether the requested force is leftward or rightward. To change force magnitude, $\Phi_{d u r}$ is modulated between 180 and 360 degrees.

\subsection{Discussion of Normal Force}

Since the ShiverPaD leverages frictional forces, the normal force between the finger and the glass can affect the production of lateral force. For example, in [20], we used a proxy finger to find that the upper bound on the lateral force production is a function normal force. The ShiverPaD device used in this paper measures the forces on real fingers rather than a proxy finger, but does so at the expense of losing the normal force information.

For simplicity, we have said lateral force is controlled, but it is probably more accurate to say that the ShiverPaD controls "normalized lateral force" $\left(F_{x} / F_{z}\right)$. We have not noticed a loss of control capability due to this difference, perhaps because people tend to scan the surface with relatively constant normal force [25].

For most of the results in this paper, normal force is irrelevant, but for the data in Fig. 7, it was important to maintain a constant normal force throughout the experiment. The data collection for this particular experiment was performed with a finger support device. It kept the finger stationary, and supported the weight of the arm and hand, making the control of normal force easier. By using the peak force values from Fig. 7, the equation developed in [20], and the coefficient of friction values found in [17], we can estimate the normal force used to be approximately $300 \mathrm{mN}$.

In an effort to wash out the normal force effects, the plotted surface in Fig. 7 is the average of several runs. While variation from run to run occurs, the standard deviation in force is less than $20 \mathrm{mN}$ over the entire $\Phi_{c e n}-\Phi_{d u r}$ domain, and the overarching shape of the surface is consistent in every trial. Some of the standard deviation data can be seen in Fig. 8b.

\subsection{Discussion of Finger Velocity}

Theoretically, as the finger's velocity approaches the ShiverPaD's peak velocity, no force can be applied in the direction of motion. If high finger exploration velocities are expected, then higher oscillation velocity will be required to achieve the target force.

Since the ShiverPaD oscillates at $854 \mathrm{~Hz}$ and $\pm 20 \mu \mathrm{m}$, the peak surface velocity is roughly $10 \mathrm{~cm} / \mathrm{s}$. In our experiments, there was no indication that finger velocity affected the perception of forces. This is probably a result of the ShiverPaD's small workspace (roughly $15 \mathrm{~mm}$ in diameter), which tended to limit finger exploration velocities. Over the course of the entire human subject trial in Section 6, the subjects' exploratory finger motion exceeded $3.0 \mathrm{~cm} / \mathrm{s}$ for only 0.4 percent of the experiment.

\section{Displaying a Toggle Switch}

Since the ShiverPaD is effectively a source of force, it is possible to display any arbitrary force field. To provide an example of this capability, we have chosen to display a virtual toggle switch.

In [20], we use $40 \mathrm{~Hz}$ shiver motion to demonstrate the implementation of line sink force fields (where all forces point toward a line) and line sources (where all forces point away from a line). On the $864 \mathrm{~Hz}$ ShiverPaD, we have created a new force field that is a collection of two line sinks and one line source (see Fig. 9a). Like a toggle switch, this field has two low potential positions with an unstable high potential position between them.

When a finger is on the surface, it tends to be pushed away from the high potential line source, and naturally gets pulled into a stable position in one of the line sinks. Therefore, when trying to move the finger back and forth smoothly, it naturally "flips" between the two low potential areas. It approximates the feel of flipping a toggle switch.

By slowly sweeping a finger back and forth across the virtual toggle switch several times, we find force as a function of position (Fig. 9b). To provide a more intuitive idea for the tactile experience, Fig. $9 c$ is the integral of the averaged force data. This integral is called the "potential function," and is defined as $V(x)=\int F(x) d x$, where $F(x)$ is the force on the finger as a function of position, $x$. In Fig. 9c, we see the two stable positions of the toggle switch as the two minimum points of the potential function (located at approximately $\pm 2 \mathrm{~mm}$ ).

\section{InTROduction to Edge Tracing ON THE SHIVERPAD}

The edges of a button give us important information about the shape and location of the button. During the exploration of a real edge, the guiding forces keeping the finger 


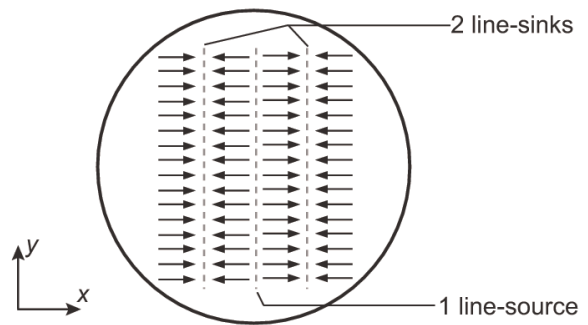

(a)

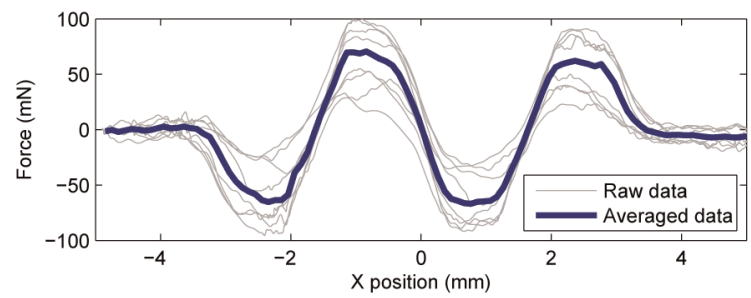

(b)

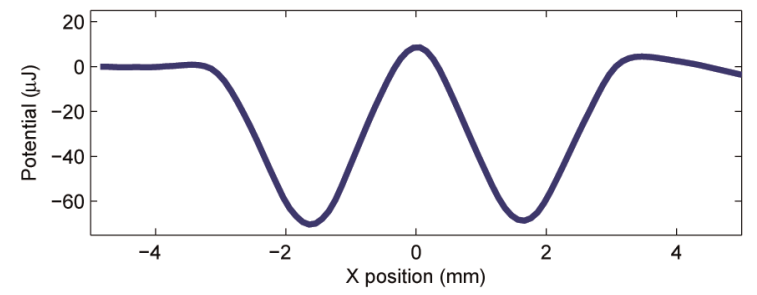

(c)

Fig. 9. Three different ways to view the force field used to display the toggle switch. (a) The toggle force field as displayed on the surface of the ShiverPaD. (b) Real force data generated by repeatedly moving a finger through the toggle force field. Four times left-to-right and four times right-to-left. (c) The potential function here is the integral of the averaged data in (b).

constrained to the edge are roughly orthogonal to the direction of travel. Since variable friction displays cannot create forces orthogonal to the direction of travel, the haptic illusion of edge following is not possible. On the other hand, the ShiverPaD is capable of creating forces orthogonal to finger motion so it has the potential to display edge-like features.

In Fig. 10, we show how two different contours (or "edges") can be displayed using the ShiverPaD. The line sink force field can also be viewed as a vertical line (Fig. 10a). Since the entire dashed line has the same low potential, it is easy to run a finger along its length. The notch contour (Fig. 10b) would likely be best displayed by creating a force field always normal to the dashed line, but this is not possible because the ShiverPaD is limited to left-right forcing directions (a limitation that future prototypes will not have). We found that lines greater than 45 degrees from the vertical did not produce convincing edge effects. For this reason, all of our contours are composed of line segments which are less than 37 degrees from the vertical.

Although the deviations in some of the contours we display on the surface of the ShiverPaD are quite small $(0.5 \mathrm{~mm})$, they can be distinguished easily because of the way the ShiverPaD displays its force. As discussed in Section 2.1.1, at any instant in time, the ShiverPaD has a constant force field across its surface. An interesting consequence to this method of force generation is that

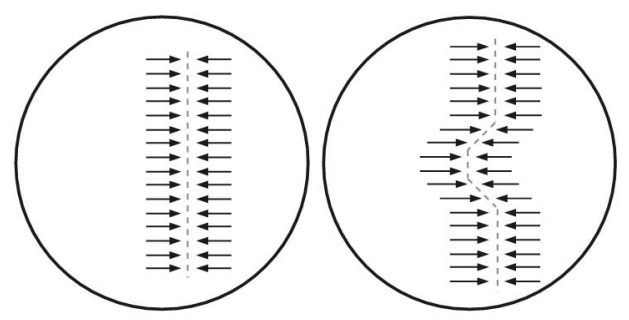

(a)

(b)

Fig. 10. The force fields used to display two edge-like contours. (a) The line contour. (b) The notch contour.

features much smaller than the finger pad are experienced across the whole finger pad. A real-life analogy to this experience is described in Fig. 11.

\section{Human Subject Trials: Detecting Edge Shape}

To demonstrate that the ShiverPaD improves edge-following capability, an experiment was conducted to show that subjects are faster at finding, and more accurate at tracing contours with the ShiverPaD when compared to the TPaD variable friction device.

\subsection{Experimental Protocol}

The protocol was reviewed and approved by Northwestern University's IRB. All subjects gave informed consent. Five subjects participated in the experiment. They were male members of the authors' lab between the ages of 19 and 25 . Three subjects had little or no experience with the ShiverPaD before the experiment and two had a moderate level of experience.

\subsubsection{The Learning Trials}

During the learning trials, contour numbers 0,4 , and 8 from Fig. 12 were displayed to the subject using both the $\mathrm{TPaD}$ and the ShiverPaD. The experimenter indicated the contour being displayed by pointing to it on a paper printout of Fig. 12. The subject explored the contour until he was confident that he could trace it. The experimenter then checked that the subject found the contour by overlaying the subject's $x-y$ finger position on the contour (creating a plot similar to those in Fig. 13). If it was clear that the subject had not successfully found the contour, the experimenter asked the subject to attempt it a second time. No subjects required a third attempt. This process was completed for both the TPaD and the ShiverPaD.

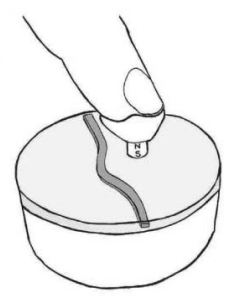

Fig. 11. The authors' interpretation of the ShiverPaD experience while edge following. Here, a magnet on the fingertip interacts with a strip of ferromagnetic material embedded below the surface of the glass. 

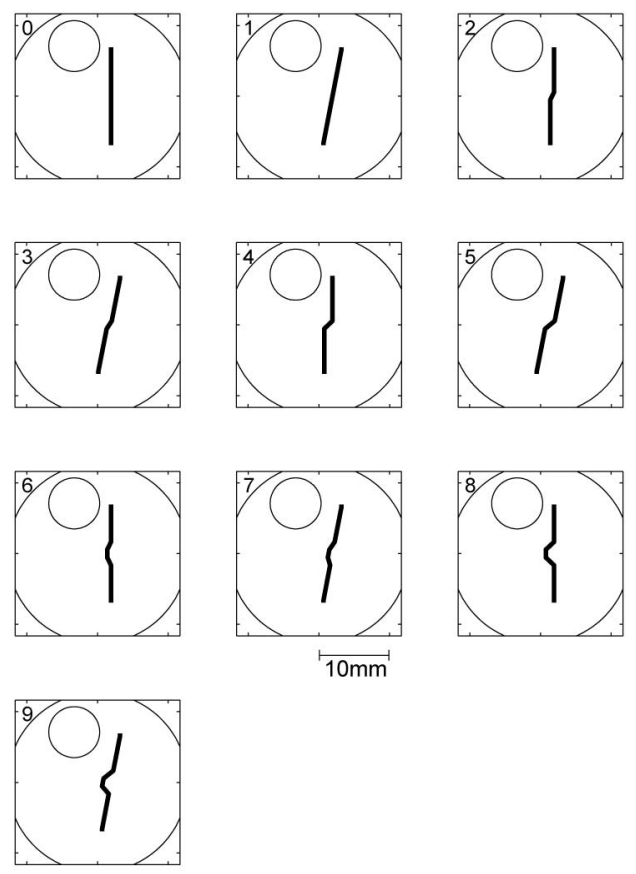

Fig. 12. The 10 contours displayed to the subject in random order. The small circle is a surface mounted sensing piezoelectric and the large circle is the edge of the TPaD.

\subsubsection{Primary Experiment}

After the learning trials, the printout of Fig. 12 was hidden from view. Each of the subjects was asked to trace the 10 contours shown in Fig. 12 using both the ShiverPaD and the $\mathrm{TPaD}$. The 10 contours were presented in randomized order. In total, 50 paired trials (100 trials) were conducted. In each pairing, the subject and contour shape were the same, but the guidance method was different (either TPaD or ShiverPaD).

Before the trials began, the subjects were given the following instructions:

1. At the beginning of each trial, you will be given a verbal indication that you can place your finger on the surface.

2. Explore the surface until you feel sufficiently able to trace the contour.

3. Trace the contour four times (e.g., trace it up-backup-back).

4. Remove your finger from the surface.

Three of the subjects were presented with the 10 ShiverPaD contours followed by the $10 \mathrm{TPaD}$ contours. The order was reversed for the other two subjects.

\subsection{Results}

It is evident from the visual inspection of the raw data, that there is an improvement in accuracy when using the ShiverPaD. Fig. 13 is the data from one of the subjects naive to the ShiverPaD.

Two metrics were used to determine the subject's ability to trace the contour.

1. $t_{\text {find }}$ (sec) is the time when the subject begins tracing the contour for the last four times before lifting his finger. The timer starts when the subject first places his finger on the surface $(t=0)$.
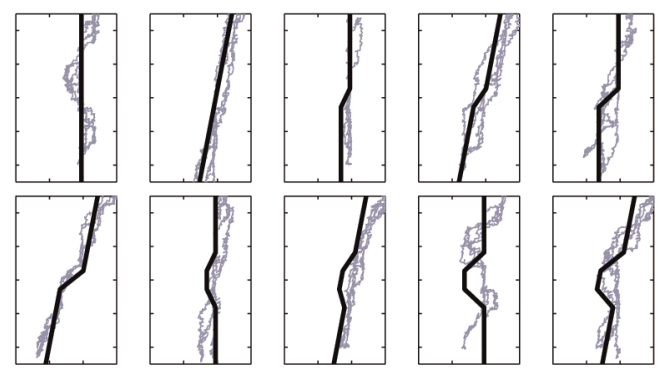

(a)
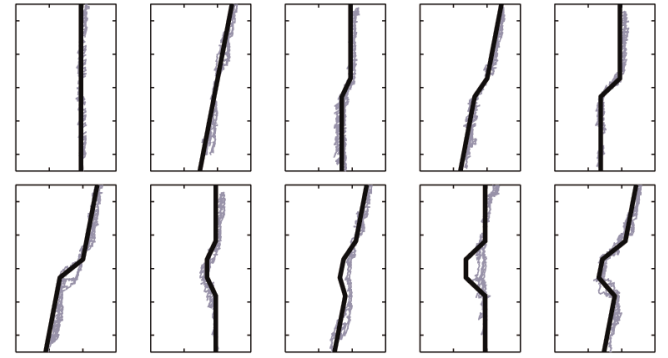

(b)
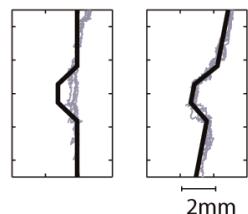

Fig. 13. Data from a single subject. The randomization has been removed for easy comparison. (a) TPaD. (b) ShiverPaD.

2. SSES $(\mathrm{mm})$ is the Square root of the Sum of the Error Squared. It is defined as $\sqrt{\left(\Sigma e_{x_{i}}^{2}\right) / n}$ where $e_{x_{i}}$ is the error in $x$-position for each time step, and $n$ is the number of time steps. It is calculated between $t_{\text {find }}$ and the time when the subject lifts his finger off of the surface.

It can be seen in the aggregate data in Fig. 14 that when using the ShiverPaD, there was a 35 percent decrease in the time to find the feature $\left(t_{\text {find }}\right)$ and a 47 percent improvement in the tracing accuracy (SSES). A paired t-test was used to find the p-values. The pairing and the calculated p-values are indicated in the figure. Their extremely low values indicate a high level of statistical significance.

To determine if the order of presentation had an effect on performance, the data were split into two groups: those who
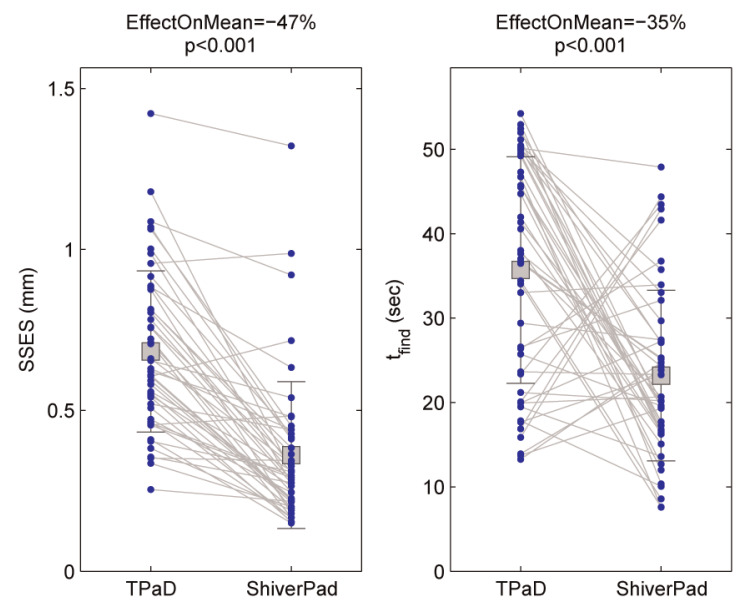

Fig. 14. The aggregate results from five subjects. The $p$-values were computing using a paired t-test. The pairings are shown by the light gray lines. The raw data, the mean, and the $\pm 1 \sigma$ are plotted. 
were presented the ShiverPaD contours first, and those who were presented the TPaD contours first. Analyzing the data in this way did not affect the conclusions. In both groups, the percentage decrease was similar to the aggregate data, and the $\mathrm{p}$-value indicated significance in all cases.

Out of the 50 paired trials, there was one ShiverPaD trial with an extremely high SSES. It was the first ShiverPaD trial for this particular subject. In the trial, the subject never found the contour and made a guess that was a long distance from the actual displayed contour. The outlier has been removed from the data in Fig. 14 leaving 49 paired trials. Inclusion of the outlier does not drastically effect the p-values.

The subjects were not explicitly asked to discuss their experience, but two of the naive subjects commented that they were very uncertain about their ability to trace the contours on the TPaD and three of the subjects commented that they thought they successfully found the ShiverPaD contours.

\section{Conclusion}

The ShiverPaD is capable of applying and controlling shear force on a finger. As with any controllable force source, it allows us to display force fields of our choosing when coupled with finger position feedback. We have demonstrated this by implementing a virtual toggle switch composed of line sources and sinks, which are perceived as 3D protrusions and depressions.

We have also demonstrated that the ShiverPaD is capable of displaying edge-like contours and that subjects naive to the device are able to locate and trace contours with only minimal training. An extension of the ShiverPaD technology could be used to display the edges of buttons on touch screens and touch pads-allowing people to find the location and shape of buttons through haptic exploration.

The concept of the ShiverPaD is easily extended to a second axis of force production by moving the TPaD in both in-plane directions. One means of achieving this is to "swirl" the TPaD in small, in-plane, circles. Since the velocity vector passes through all 360 degrees during the swirl, it is possible to create forces in any in-plane direction. Work on a two-axis version, called the SwirlPaD, has already produced forces in both in-plane directions.

Other future work for ShiverPaD technology includes,

- Creating larger TPaD surfaces.

- Reducing audible noise.

- Further reducing the perception of vibration.

- Increasing force magnitude.

There are still challenges, but our ShiverPaD prototype is a significant step toward a visually transparent haptic surface capable of applying any arbitrary shear force to a finger. This device would have the capability of displaying 2D worlds composed of springs, dampers, masses, and force fields; it would enable the creation of illusory 3D features, like bumps and toggle switches; and allow the user to trace the virtual edges of buttons and other features.

\section{ACKNOWLEDGMENTS}

The authors would like to acknowledge the US National Science Foundation (NSF) (grant IIS-0941581) and a gift from Research in Motion Ltd for support of this work. Thanks go to Daniel C. Johnson for his help in the design and construction of the finger positioning system.

\section{REFERENCES}

[1] S.-C. Kim, T.-H. Yang, B.-K. Han, and D.-S. Kwon, "Interaction with a Display Panel-An Evaluation of Surface-Transmitted Haptic Feedback," Proc. Int'l Conf. Control, Automation, and Systems, Oct. 2008.

[2] I. Poupyrev, S. Maruyama, and J. Rekimoto, "Ambient Touch: Designing Tactile Interfaces for Handheld Devices," Proc. 15th Ann. ACM Symp. User Interface Software and Technology, pp. 51-60, 2002.

[3] I. Oakley, J. Ängeslevä, S. Hughes, and S. OModhrain, "Tilt and Feel: Scrolling with Vibrotactile Display," Proc. EuroHaptics, June 2004.

[4] I. Poupyrev and S. Maruyama, "Tactile Interfaces for Small Touch Screens," Proc. 16th Ann. ACM Symp. User Interface Software and Technology, pp. 217-220, 2003.

[5] ArtificialMuscle, "Smarttouch Technology,"www.artificialmuscle. com, 2009.

[6] G.-H. Yang, K.-U. Kyung, M. Srinivasan, and D.-S. Kwon, "Quantitative Tactile Display Device with Pin-Array Type Tactile Feedback and Thermal Feedback," Proc. IEEE Int'l Conf. Robotics and Automation, pp. 3917-3922, May 2006.

[7] Y. Kato, T. Sekitani, M. Takamiya, M. Doi, K. Asaka, T. Sakurai, and T. Someya, "Sheet-Type Braille Displays by Integrating Organic Field-Effect Transistors and Polymeric Actuators," IEEE Trans. Electron Devices, vol. 54, no. 2, pp. 202-209, Feb. 2007.

[8] J. Pasquero and V. Hayward, "Stress: A Practical Tactile Display with One Millimeter Spatial Resolution and $700 \mathrm{~Hz}$ Refresh Rate," Proc. EuroHaptics, July 2003.

[9] V. Vincent Levesque and V. Hayward, "Experimental Evidence of Lateral Skin Strain During Tactile Exploration," Proc. EuroHaptics, July 2003.

[10] C. Harrison and S.E. Hudson, "Providing Dynamically Changeable Physical Buttons on a Visual Display," Proc. 27th Int'l Conf. Human Factors in Computing Systems, pp. 299-308, 2009.

[11] M.D.R.R. Minsky, "Computational Haptics: The Sandpaper System for Synthesizing Texture for a Force-Feedback Display," PhD dissertation, Massachusetts Inst. of Technology, 1995.

[12] G. Robles-De-La-Torre, "Comparing the Role of Lateral Force During Active and Passive Touch: Lateral Force and its Correlates are Inherently Ambiguous Cues for Shape Perception under Passive Touch Conditions," Proc. EuroHaptics, pp. 159-164, 2002.

[13] A. Yamamoto, T. Ishii, and T. Higuchi, "Electrostatic Tactile Display for Presenting Surface Roughness Sensation," Proc. IEEE Int'l Conf. Industrial Technology, pp. 680-684, Dec. 2003.

[14] M. Takasaki, H. Kotani, T. Mizuno, and T. Nara, "Transparent Surface Acoustic Wave Tactile Display," Proc. IEEE/RSJ Int'l Conf. Intelligent Robots and Systems, pp. 3354-3359, Aug. 2005.

[15] T. Watanabe and S. Fukui, "A Method for Controlling Tactile Sensation of Surface Roughness Using Ultrasonic Vibration," Proc. IEEE Int'l Conf. Robotics and Automation, vol. 1, pp. 1134-1139, May 1995.

[16] M. Biet, F. Giraud, and B. Lemaire-Semail, "Implementation of Tactile Feedback by Modifying the Perceived Friction," The European Physical J. Applied Physics, vol. 43, no. 1, pp. 123-135, July 2008.

[17] L. Winfield, J. Glassmire, J.E. Colgate, and M. Peshkin, "T-PaD: Tactile Pattern Display through Variable Friction Reduction," Proc. Second Joint EuroHaptics Conference and Symp. Haptic Interfaces for Virtual Environment and Teleoperator Systems, World Haptics Conf., pp. 421-426, 2007.

[18] D. Wang, K. Tuer, M. Rossi, and J. Shu, "Haptic Overlay Device for Flat Panel Touch Displays," Proc. 12th Int'l Symp. Haptic Interfaces for Virtual Environment and Teleoperator Systems, 2004.

[19] D. Wang, M. Rossi, K. Tuer, and D. Madill, "Method and System for Providing Haptic Effects," US Patent Application Publication, no. 20060209037, Sept. 2006.

[20] E.C. Chubb, J.E. Colgate, and M.A. Peshkin, "ShiverPaD: A Device Capable of Controlling Shear Force on a Bare Finger," Proc. Third Joint EuroHaptics Conference and Symp. Haptic Interfaces for Virtual Environment and Teleoperator Systems, World Haptics Conf., pp. 1823, 2009.

[21] S.J. Bolanowski Jr., G. Gescheider, R. Verrillo, and C. Checkosky, "Four Channels Mediate the Mechanical Aspects of Touch," The J. Acoustical Soc. of Am., vol. 84, no. 5, pp. 1680-1694, 1988. 
[22] S.O.R. Moheimani, "A Survey of Recent Innovations in Vibration Damping and Control Using Shunted Piezoelectric Transducers," IEEE Trans. Control Systems Technology, vol. 11, no. 4, pp. 482-494, July 2003.

[23] N.W. Hagood and A.V. Flotow, "Damping of Structural Vibrations with Piezoelectric Materials and Passive Electrical Networks," J. Sound and Vibration, vol. 146, no. 2, pp. 243-268, 1991.

[24] E.C. Chubb, "Shiverpad: A Haptic Surface Capable of Applying Shear Forces to Bare Finger," master's thesis, Northwestern Univ., 2009.

[25] S. Choi, L. Walker, H.Z. Tan, S. Crittenden, and R. Reifenberger "Force Constancy and Its Effect on Haptic Perception of Virtual Surfaces," ACM Trans. Applied Perceptions, vol. 2, no. 2, pp. 89-105, 2005.

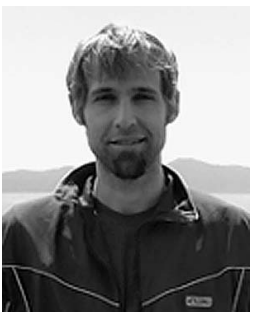

Erik C. Chubb received the BSME degree from the University of Michigan and the MSME degree from Northwestern University in 2009. He wrote Electronic Stability Control software for Ford Motor Company and holds several patents and a Henry Ford Technology award. His interests include haptics, modeling and controls, optimization techniques, and clean energy. $\mathrm{He}$ is a student member of the IEEE.

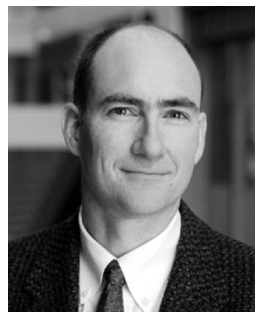

J. Edward Colgate is a professor in the Department of Mechanical Engineering. His principal research interest is human-robot interaction. He has worked extensively in the areas of haptic interface and teleoperation, and he, along with collaborator Michael Peshkin, is the inventor of a class of collaborative robots known as cobots. In addition to his academic pursuits, he is a founder of Stanley Cobotics, the leading supplier of intelligent ergonomic assist devices to the industrial marketplace and of Kinea Design, a start-up devoted to rehabilitation robotics and advanced mechatronics. He is a member of the IEEE.

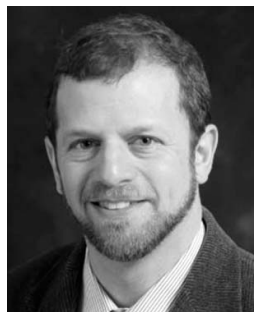

Michael A. Peshkin received the PhD degree from MIT. He is a professor of mechanical engineering in the Department of Mechanical Engineering, Northwestern University. His research is in robotics and human-machine interaction, and rehabilitation robotics. He also works in novel actuators and sensors. He has cofounded three spin-offs: Mako Surgical (image guided surgery), Cobotics (assistive devices for materials handling), and Kinea Design (gait and balance training robot for rehabilitation after stroke). He holds 15 patents and is a coinventor (with J. Edward Colgate) of Cobots: collaborative robots for direct interaction with humans in a shared task. He is a member of the IEEE.

$\triangleright$ For more information on this or any other computing topic, please visit our Digital Library at www.computer.org/publications/dlib. 